\title{
An efficient method to find potentially universal population genetic markers, applied to metazoans
}

\author{
Anne Chenuil ${ }^{11^{*}}$, Thierry B Hoareau ${ }^{1}$, Emilie Egea ${ }^{11}$, Gwilherm Penant $^{11}$, Caroline Rocher ${ }^{11}$, Didier Aurelle ${ }^{11}$, \\ Kenza Mokhtar-Jamai ${ }^{11}$, John DD Bishop ${ }^{2}$, Emilie Boissin ${ }^{3}$, Angie Diaz ${ }^{4}$, Manuela Krakau ${ }^{5}$, \\ Pieternella C Luttikhuizen ${ }^{6}$, Francesco P Patti ${ }^{7}$, Nicolas Blavet ${ }^{8}$, Sylvain Mousset ${ }^{9,10}$
}

\begin{abstract}
Background: Despite the impressive growth of sequence databases, the limited availability of nuclear markers that are sufficiently polymorphic for population genetics and phylogeography and applicable across various phyla restricts many potential studies, particularly in non-model organisms. Numerous introns have invariant positions among kingdoms, providing a potential source for such markers. Unfortunately, most of the few known EPIC (Exon Primed Intron Crossing) loci are restricted to vertebrates or belong to multigenic families.

Results: In order to develop markers with broad applicability, we designed a bioinformatic approach aimed at avoiding multigenic families while identifying intron positions conserved across metazoan phyla. We developed a program facilitating the identification of EPIC loci which allowed slight variation in intron position. From the Homolens databases we selected 29 gene families which contained 52 promising introns for which we designed 93 primer pairs. PCR tests were performed on several ascidians, echinoderms, bivalves and cnidarians. On average, 24 different introns per genus were amplified in bilaterians. Remarkably, five of the introns successfully amplified in all of the metazoan genera tested (a dozen genera, including cnidarians). The influence of several factors on amplification success was investigated. Success rate was not related to the phylogenetic relatedness of a taxon to the groups that most influenced primer design, showing that these EPIC markers are extremely conserved in animals.
\end{abstract}

Conclusions: Our new method now makes it possible to (i) rapidly isolate a set of EPIC markers for any phylum, even outside the animal kingdom, and thus, (ii) compare genetic diversity at potentially homologous polymorphic loci between divergent taxa.

\section{Background}

Despite the crucial need for genetic markers independent from the non recombining mitochondrial genome, nuclear markers remain much less used than mitochondrial ones in metazoans (in the Entrez-Nucleotide database, there are about ten times fewer entries containing "population" and "nuclear marker" or "population" and "nuclear", when used as key-words, than entries containing "mitochondrial" instead of "nuclear"). When

\footnotetext{
* Correspondence: anne.chenuil-maurel@univmed.fr

${ }^{11}$ Aix-Marseille Université, Laboratoire DIMAR (diversité, évolution et écologie fonctionnelle marine), CNRS UMR6540, rue de la batterie des Lions, 13007 Marseille, France

Full list of author information is available at the end of the article
}

choosing molecular genetic markers for a given biodiversity study, two properties, codominance and the possibility of reconstructing evolutionary relationships among alleles, are generally desirable but are often difficult to obtain [1]. During the last decade, microsatellites became the most popular codominant markers. However, introns are well known for providing potential markers variable within species, using EPIC-PCR. EPIC loci have several advantages compared to microsatellites. Owing to the position of the primers in conserved exons, EPICs are potentially applicable across species and much less prone to null alleles. After sequencing the variants, evolutionary relationships among alleles can be inferred much more accurately than for 
microsatellites, which are very susceptible to homoplasy [2]. There is also a less well known but important problem with microsatellites: in some species, most microsatellites appear to belong to the same family (or a small number of families) of repeated elements; in such cases codominant genotyping is difficult since the primers often anneal to multiple paralogous regions $([3,4]$ and Chenuil, unpublished).

A recent computer program [5] allows the identification of introns at conserved positions in a species for which EST sequences are available by comparison with a related model species for which extensive sequence information is available (see also [6]). Another study developed a bioinformatic pipeline to identify EPIC loci by comparison of two or more whole-genome sequence datasets, and tested a dozen of these loci by PCR-sequencing in distantly related teleost fishes [7]. By contrast, our approach is designed to find intron positions and define primers able to amplify a wider variety of species from which we may have absolutely no sequence data.

The positions of introns are extremely conserved during evolution; for instance, $14 \%$ of animal introns match plant intron positions $[8,9]$. Although this should have favoured the development of EPIC markers conserved in different phyla, only half a dozen EPIC loci were proposed by [10] and by [11]. These loci have rarely been used outside vertebrates (e.g. [12-14]) and in numerous species none of the tested EPIC loci appeared usable (e.g. [15]). EPIC loci can be developed specifically for a given taxon, often using genomic and cDNA sequence data (e.g. [16,17]; Aurelle et al., submitted). Only vertebrates benefit from a relatively consistent set of EPIC loci $[18,19]$. Several reasons for such biases can be invoked. (1) These "universal" EPIC loci were chosen in extremely conserved genes, and, not surprisingly, often appeared to belong to multigenic families, which limited their use as codominant markers due to simultaneous amplification of paralogs. (2) Few sequences were available (often none outside vertebrates) to properly define PCR primers, thus PCR amplifications often failed. (3) Primers were generally designed considering the nucleotide variation observed in the data set available (often phylogenetically limited), but ignoring amino-acid conservation and code degeneracy.

Our study was designed to avoid these shortcomings. We identified putative universal EPIC loci and designed primers to amplify them in metazoans, taking advantage of the increased availability of properly annotated, phylogenetically diverse whole-genome sequences. We actively avoided multigenic families, and used a primer design strategy aimed at preserving amino-acid sequences while allowing synonymous codon changes. For this purpose, we developed dedicated bioinformatic tools, and then tested all the primer pairs designed in divergent animal phyla for PCR amplification under relatively standardized conditions.

\section{Results-Discussion}

\section{Potential EPIC loci found in data bases}

The number of introns for which we designed and tested primer pairs was respectively 15 (named between i1 to i17), 11 (i19 to i35) and 22 (i36 to i58) for stages I, II and III described in the Methods section (Fig. 1, Fig. 2 ). Intron numbers are not consecutive because we could not design satisfactory primer pairs for some introns that appeared good at the preceding step. In addition, we designed primers for two genes containing already known "universal" EPICs: in ATPS- $\alpha$, one intron corresponds to the one in Jarman et al (2002) [11] with slightly different primer sequences, and primers were designed for an additional intron; in EF1- $\alpha$, two primer pairs were designed for each of two introns. The 52 introns came from 29 different gene families and corresponded to 93 primer pairs tested in all species. Surprisingly, a single family was retained from both stages I and II (so that intron 2 is the same as intron 22). From the 89 families containing no duplication nodes (i.e. a node containing the same clusters of taxa several times, due to gene duplication) isolated during stage I, only six introns appeared to be present at exactly the same nucleotide position in $100 \%$ of the species, and for only 3 of these, corresponding to EPIC 1, 4 and 5, could we design satisfactory primer pairs (Fig. 1). Thus, we also designed primers for some introns that were present in $100 \%$ of the deuterostome species in the amino-acid alignments (i.e. vertebrates and Ciona, no echinoderm species being available in the Homolens database). For subsequent stages, intron position was not necessarily perfectly invariant (see Methods). Fig. 3 displays an example of the output of the graphical script designed for stage III. For 36 introns (56 primer pairs out of 93) we could define a forward and a reverse primer whose degeneracy did not exceed 8-fold. For 12 of them (22 primer pairs), degeneracy was lower than or equal to 6 . In particular, the primers designed for introns 21,25 , $26,45,50$ and 54 displayed no more than a 2- or 4-fold degeneracy (Fig. 1).

\section{Effect of taxonomy on PCR results}

For each stage, protein family, and intron, Table 1 and Table 2 report the results of the PCR for each genus as observed on agarose gels (Fig. 4) (and the total number of genera), distinguishing the three quality levels ' $\mathrm{P}$ '(Promising), 'I' (Intron-size amplicon) and 'A' (Amplification) defined in the Methods section V. The number of introns producing an amplification product large enough to contain an intron of at least $70 \mathrm{bp}$, i.e. pooling the 

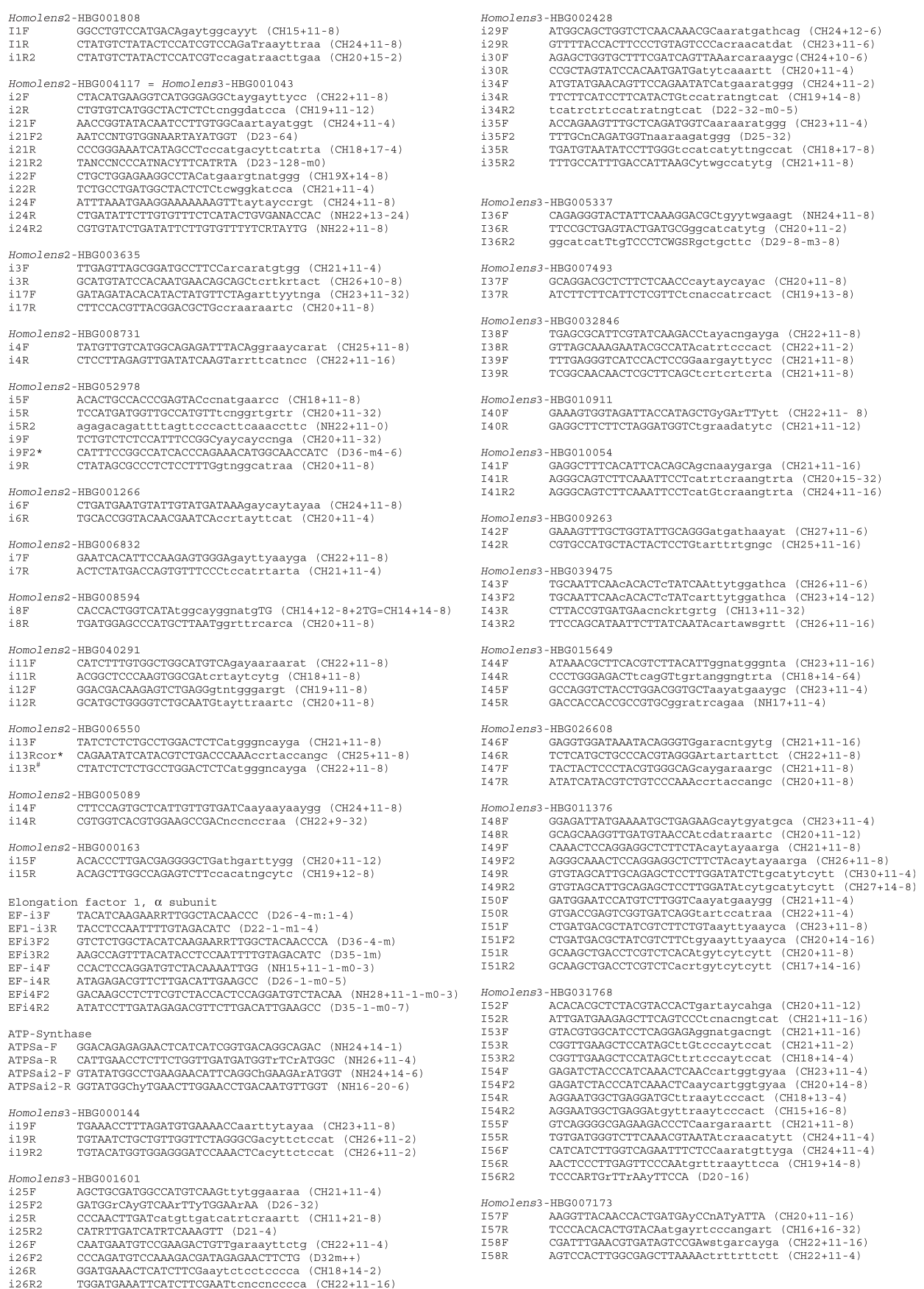

Figure 1 Primer sequences. Sequences are written using the IUPAC code. We used the following codes to describe the rules that underpinned primer design: Capital letters represent the $5^{\prime} \mathrm{clamp}$ (non-degenerate), whereas small letters represent the degenerate part supposed to contain no mismatch whatever the species (based on known sequences, protein for $\mathrm{CH}$ primers or nucleotide for $\mathrm{D}$ or $\mathrm{NH}$ primers). $\mathrm{CHX}+\mathrm{Y}-\mathrm{Z}$ : CodeHop primer with a $5^{\prime}$ clamp (non-degenerate part) of $X$ bases, and a $3^{\prime} Z$-fold degenerate end of $Y$ bases. $C H X+Y-Z+2 G T$ : CodeHop primer designed at the intron limit, which contains the first two bases of the intron (by mistake, we reversed the two bases in the single such case, i8F). DX-Ymz-t: Classical degenerate primer of $X$ bases long, $Y$-fold degenerate, containing $z$ to $t$ mismatches according to the species (despite degeneracy in primer design). NHX+Y-Z: We called this a 'Nucleotide-hop' primer, by homology with CodeHop primers, but design was based on nucleotide alignment; we designed a $5^{\prime}$ clamp (non-degenerate) and degenerate the $3^{\prime}$ end according to the set of nucleotide sequences available thus ignoring codons. NHX+Y-Z-mz-t: Same as above, but, despite primer degeneracy, there may remain mismatches in some species; in this case there are from $z$ to $t$ mismatches according to the species for which we have sequence data. For instance, a primer (D30-1-m0-2) actually does not contain ambiguity bases (-1: not degenerate), and contains 0 to 2 mismatches according to the species. Other symbols: ${ }^{*}$ this primer was not used. \# erroneous primer sequence, the subsequently corrected primer i13Rcor was not tried 


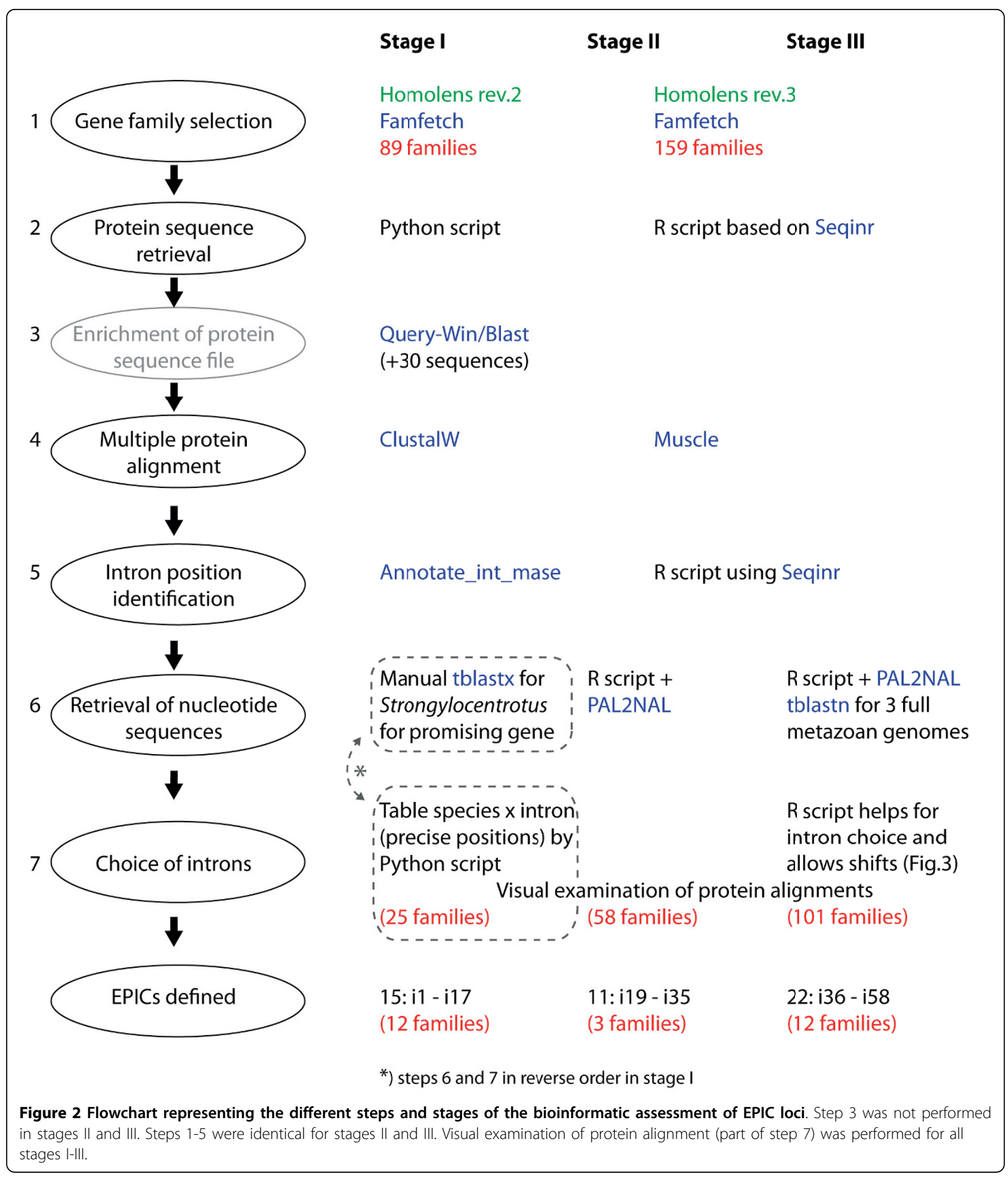

'P' + 'I' categories, is rather similar among genera, and varies from 20 to 30 introns (out of 51 tested), i.e. from about $40 \%$ to $60 \%$ of the introns. Some of the factors likely to generate variation in this value among taxa are: (i) the number of PCR conditions tested among genera, (ii) DNA extract quality, (iii) phylogenetic distance of the taxon from Strongylocentrotus and Ciona, the species which most influenced primer design, (iv) the molecular evolutionary rate of the phylum or lineage, and (v) the average size of introns in the taxon (some taxa tend to 


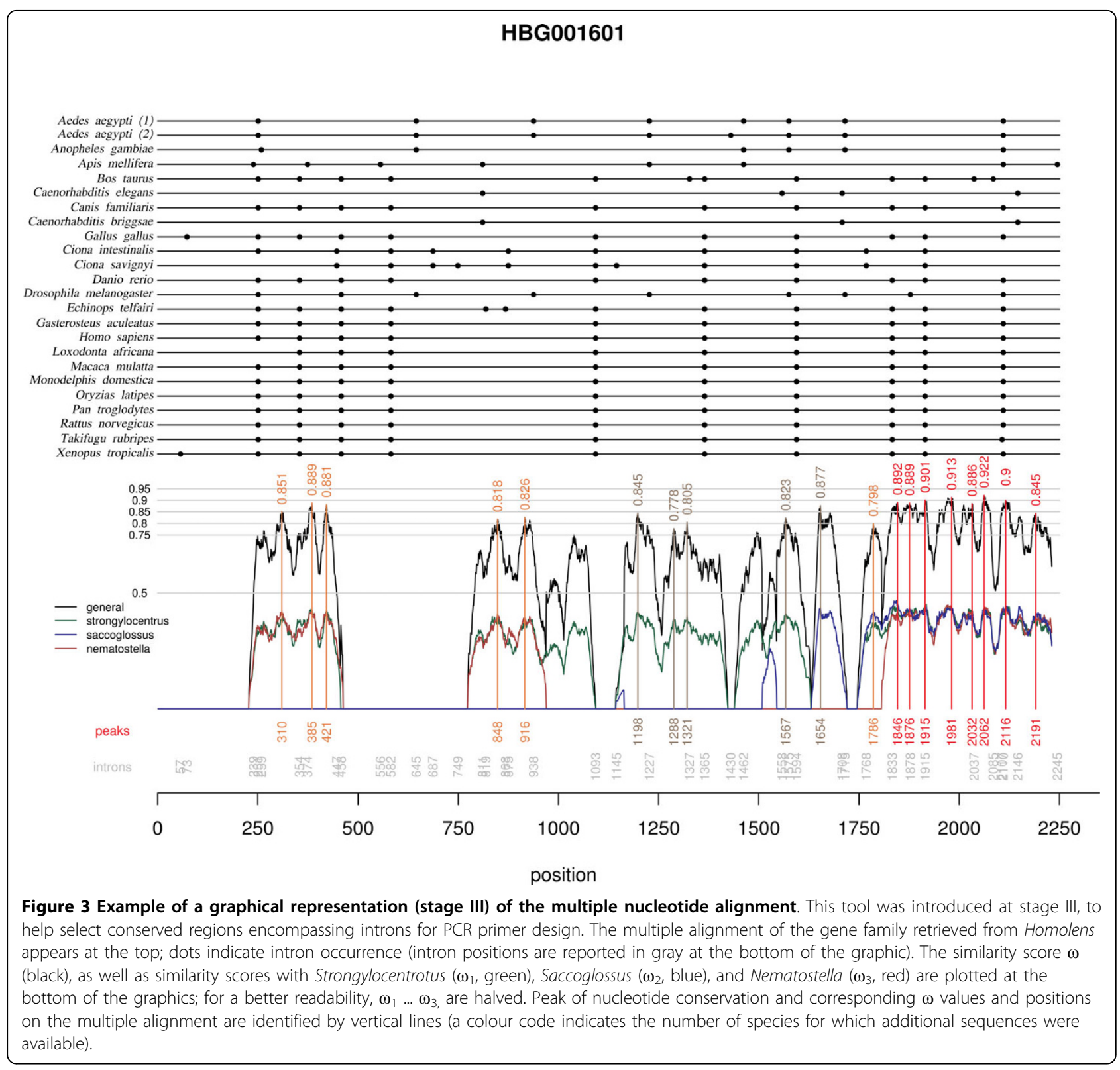

have large, thus not amplifiable introns, or too small introns eventually recorded as 'A' despite the presence of an intron). The number of "promising" introns ('P') appears more variable among taxa (from 6 to 15 introns, i.e. $12 \%$ to $29 \%$ ). Factors causing variation in the proportion of "promising" introns (and which should not necessarily affect the number of ' $\mathrm{P}+\mathrm{I}$ ' introns) may be: (i) quality of DNA extracts (see below, section 5) since null PCR may infrequently occur due to bad DNA extracts and cause a locus in a genus be scored as ' $\mathrm{I}$ ' instead of ' $\mathrm{P}$ ' (even if a single individual of the 4 extracts tested does not amplify), (ii) variable tendency to display null alleles (e.g. alleles producing no amplification products) among taxa (a consequence of intrinsic polymorphism or effective population sizes), and (iii) genomic features such as the ploidy level or the frequency of transposable elements (causing multiple band patterns, for instance).

Surprisingly (Fig. 5) we did not observe any clear relationship between phylogeny and global PCR success (i.e. number of successful loci per genus, whatever the quality level considered as successful), with an average of 22 , 23 and 25 introns amplifying (I or P) per genus in bivalves, ascidians and echinoderms respectively (about 10-12 of which yielded promising 'P' patterns in each group). Some genera belonging to protostome phyla displayed better results than some deuterostomes. The cnidarian Corallium, in which only 29 loci instead of 
Table 1 Results of PCR amplification for each intron locus in each genus

\begin{tabular}{|c|c|c|c|c|c|c|c|c|c|c|c|c|c|}
\hline Intron & 1 & 2 & 3 & 4 & 5 & 6 & 7 & 8 & 9 & 11 & 12 & 13 & 14 \\
\hline Homolens version & $\|$ & $\|$ & $\|$ & $\|$ & $\|$ & $\|$ & $\|$ & $\|$ & $\|$ & $\|$ & $\|$ & $\|$ & $\|$ \\
\hline Gene family (HBG-code) & 004117 & 001043 & 003635 & 008731 & 052978 & 001266 & 006832 & 008594 & 052978 & 040291 & 040291 & 006550 & 005089 \\
\hline $\begin{array}{l}\text { No primer pairs tested } \\
\text { (without errors) }\end{array}$ & 2 & 1 & 1 & 1 & 2 & 1 & 1 & 1 & 1 & 1 & 1 & 1 & 1 \\
\hline $\begin{array}{l}\text { PCR size expected if intron } \\
\text { absent }\end{array}$ & 174 & 100 & 80 & $?$ & $90-130$ & 75 & 60 & 110 & 90 & 70 & 150 & 170 & 110 \\
\hline No genera ' $P$ ' & 6 & 8 & 0 & 0 & 8 & 0 & 0 & 0 & 3 & 1 & 1 & 0 & 0 \\
\hline No genera 'l' & 3 & 2 & 4 & 0 & 2 & 0 & 1 & 4 & 5 & 7 & 7 & 1 & 1 \\
\hline No genera ' $A$ ' & 1 & 0 & 0 & 0 & 0 & 0 & 0 & 0 & 1 & 0 & 0 & 0 & 0 \\
\hline Paracentrotus & 1 & $P$ & & & $P$ & & & & $P$ & 1 & I & & \\
\hline Amphipholis & $P$ & $P$ & 1 & & $P$ & & I & & $P$ & । & & & । \\
\hline Echinocardium (all conditions) & $P$ & $P$ & । & & $P$ & & & & & । & $P$ & & \\
\hline Corella & A & $P$ & । & & $P$ & & & & । & । & । & & \\
\hline Perophora & $P$ & $P$ & & & $P$ & & & & । & $P$ & & & \\
\hline Styela & $P$ & $P$ & & & $P$ & & & & $P$ & । & I & & \\
\hline Abatus (all species) & । & $P$ & & & $P$ & & & I & I & & I & I & \\
\hline Sterechinus (all species) & $P$ & $P$ & & & I & & & I & 1 & & I & & \\
\hline Macoma & 1 & 1 & । & & I & & & I & I & 1 & I & & \\
\hline Cerastoderma & $P$ & । & & & $P$ & & & । & A & । & । & & \\
\hline Corallium & $P$ & 1 & na & na & I & na & na & na & 1 & na & na & na & na \\
\hline Paramuricea & & na & na & na & 1 & na & na & na & na & na & na & na & na \\
\hline Intron & 15 & 17 & 19 & 21 & 22 & 24 & 25 & 26 & 29 & 30 & 34 & 35 & 36 \\
\hline Homolens version & $\|$ & $\|$ & III & III & III & III & III & III & III & III & III & III & III \\
\hline Gene family (HBG-code) & 000163 & 003635 & 000144 & 001043 & 001043 & 001043 & 001601 & 001601 & 002428 & 002428 & 002428 & 002428 & 005337 \\
\hline $\begin{array}{l}\text { No primer pairs tested } \\
\text { (without errors) }\end{array}$ & 1 & 1 & 2 & 4 & 1 & 2 & 4 & 4 & 1 & 1 & 2 & 4 & 2 \\
\hline $\begin{array}{l}\text { PCR size expected if intron } \\
\text { absent }\end{array}$ & 170 & 135 & $\begin{array}{l}120- \\
135 \\
\end{array}$ & 120 & 150 & 180 & $\begin{array}{l}193- \\
210 \\
\end{array}$ & 180 & 165 & 155 & $155-165$ & $\begin{array}{r}120- \\
155 \\
\end{array}$ & $\begin{array}{c}125- \\
145 \\
\end{array}$ \\
\hline No genera 'P' & 3 & 0 & 2 & 5 & 2 & 0 & 5 & 0 & 5 & 2 & 3 & 0 & 4 \\
\hline No genera 'l' & 6 & 0 & 0 & 5 & 3 & 0 & 5 & 1 & 3 & 2 & 2 & 0 & 4 \\
\hline No genera ' $A$ ' & 1 & 0 & 4 & 0 & 3 & 0 & 0 & 0 & 0 & 6 & 1 & 0 & 0 \\
\hline Paracentrotus & 1 & & $P$ & $P$ & $P$ & & I & & $P$ & $P$ & $P$ & & \\
\hline Amphipholis & 1 & & & 1 & 1 & & I & & I & A & & & I \\
\hline Echinocardium (all conditions) & 1 & & & $P$ & $P$ & & । & & $P$ & । & & & \\
\hline Corella & 1 & & & । & । & & $P$ & & । & A & & & I \\
\hline Perophora & $P$ & & $P$ & $P$ & & & $P$ & & & A & & & $P$ \\
\hline Styela & 1 & & & I & & & $P$ & & I & 1 & A & & $P$ \\
\hline Abatus (all apecies) & $P$ & & A & $P$ & A & & I & & $P$ & $P$ & $P$ & & $P$ \\
\hline Sterechinus (all species) & A & & A & $P$ & । & & $P$ & & $P$ & A & । & & । \\
\hline Macoma & । & & A & । & A & & $P$ & I & $P$ & A & 1 & & । \\
\hline Cerastoderma & $P$ & & A & । & A & & I & & & A & $P$ & & $\mathrm{P}$ \\
\hline Corallium & 1 & na & na & । & 1 & na & $P$ & na & 1 & na & na & na & \\
\hline Paramuricea & 1 & na & na & na & na & na & na & na & na & na & na & na & \\
\hline Intron & 37 & 38 & 39 & 40 & 41 & 42 & 43 & 44 & 45 & 46 & 47 & 48 & 49 \\
\hline Homolens version & III & III & III & III & III & III & III & III & III & III & III & III & III \\
\hline Gene family (HBG-code) & 007493 & 032846 & 032846 & 010911 & 010054 & 009263 & 039475 & 015649 & 015649 & 026608 & 026608 & 011376 & 011376 \\
\hline $\begin{array}{l}\text { No primer pairs tested } \\
\text { (without errors) }\end{array}$ & 1 & 1 & 1 & 1 & 2 & 1 & 4 & 1 & 1 & 1 & 1 & 1 & 4 \\
\hline $\begin{array}{l}\text { PCR size expected if intron } \\
\text { absent }\end{array}$ & 125 & 160 & 95 & 180 & 255 & 140 & $\begin{array}{l}110- \\
145 \\
\end{array}$ & 135 & 150 & 75 & 215 & 285 & $\begin{array}{l}240- \\
260 \\
\end{array}$ \\
\hline No genera ' $P$ ' & 4 & 1 & 2 & 0 & 0 & 0 & 5 & 1 & 1 & 1 & 0 & 4 & 0 \\
\hline
\end{tabular}


Table 1 Results of PCR amplification for each intron locus in each genus (Continued)

\begin{tabular}{|c|c|c|c|c|c|c|c|c|c|c|c|c|c|}
\hline No genera 'I' & 2 & 2 & 0 & 0 & 2 & 4 & 2 & 1 & 4 & 3 & 1 & 5 & 0 \\
\hline No genera ' $A$ ' & 0 & 1 & 0 & 0 & 0 & 0 & 0 & 3 & 0 & 0 & 2 & 0 & 0 \\
\hline Paracentrotus & & A & $P$ & & I & & & A & & I & & & \\
\hline Amphipholis & I & । & & & & 1 & I & । & & । & । & $P$ & \\
\hline Echinocardium (all conditions) & $P$ & & $P$ & & & I & $\mathrm{P}$ & A & & & & I & \\
\hline Corella & & & & & & I & $P$ & $P$ & I & & & 1 & \\
\hline Perophora & $P$ & I & & & & & P & A & P & । & A & । & \\
\hline Styela & $P$ & $P$ & & & & । & I & & I & & A & $P$ & \\
\hline Abatus (all species) & I & & & & I & & $P$ & & & $P$ & & $P$ & \\
\hline Sterechinus (all species) & & & & & & & & & & & & $P$ & \\
\hline Macoma & & & & & & & $P$ & & I & & & I & \\
\hline Cerastoderma & $P$ & & & & & & & & I & & & I & \\
\hline Corallium & A & 1 & & & na & & $P$ & na & I & $P$ & I & & A \\
\hline Paramuricea & 1 & 1 & & & na & & I & na & & 1 & 1 & & \\
\hline Intron & 50 & 51 & 52 & 53 & 54 & 55 & 56 & 57 & 58 & ATPs $\alpha$ J & ATPS $\alpha i 2$ & EF3 & EF4 \\
\hline Homolens version & III & III & III & III & III & III & III & III & III & & & & \\
\hline Gene family (HBG-code) & 011376 & 011376 & 031768 & 031768 & 031768 & 031768 & 031768 & 007173 & 007173 & & & & \\
\hline $\begin{array}{l}\text { No primer pairs tested } \\
\text { (without errors) }\end{array}$ & 1 & 4 & 1 & 2 & 4 & 1 & 2 & 1 & 1 & 1 & 1 & 4 & 4 \\
\hline $\begin{array}{l}\text { PCR size expected if intron } \\
\text { absent }\end{array}$ & 120 & 240 & 260 & 120 & 75 & 160 & 260 & 145 & 210 & 90 & 330 & $\begin{array}{l}250- \\
280\end{array}$ & $210-35$ \\
\hline No genera ' $P$ ' & 6 & 5 & 1 & 5 & 3 & 1 & 2 & 2 & 0 & 2 & 2 & 1 & 3 \\
\hline No genera 'l' & 4 & 4 & 0 & 2 & 4 & 1 & 2 & 1 & 1 & 4 & 3 & 4 & 6 \\
\hline No genera ' $A$ ' & 0 & 0 & 0 & 0 & 1 & 0 & 0 & 0 & 0 & 0 & 0 & 4 & 1 \\
\hline Paracentrotus & $P$ & $P$ & $P$ & $P$ & $P$ & & & I & & & & A & I \\
\hline Amphipholis & P & 1 & & । & $P$ & & & & & I & I & I & 1 \\
\hline Echinocardium (all conditions) & । & & & $P$ & $P$ & $P$ & & & & । & I & & 1 \\
\hline Corella & $P$ & 1 & & & A & & $\mathrm{P}$ & & & । & & । & A \\
\hline Perophora & $P$ & $P$ & & & । & & । & & & । & & $P$ & 1 \\
\hline Styela & 1 & I & & & 1 & & 1 & & & $P$ & & A & 1 \\
\hline Abatus (all species) & । & $P$ & & $P$ & I & । & & $P$ & & & । & I & I \\
\hline Sterechinus (all species) & $P$ & $P$ & & $P$ & I & & & $P$ & & & $P$ & 1 & $P$ \\
\hline Macoma & $P$ & 1 & & । & & & & & । & $P$ & & A & $P$ \\
\hline Cerastoderma & 1 & $P$ & & $P$ & & & $P$ & & & & $P$ & $A$ & $P$ \\
\hline Corallium & I & $P$ & A & P & I & A & A & & na & na & na & na & na \\
\hline Paramuricea & $P$ & & na & P & I & & P & P & na & na & na & na & na \\
\hline
\end{tabular}

Empty cells correspond to absence of amplification product or occurrence of primer dimer; "na" (not available) corresponds to introns not tested in the considered genus

52 were surveyed, provided 18 amplifying introns (I + $\mathrm{P})$. This absence of detectable phylogenetic influence is probably not an artefact resulting from variation in technical effort since, even when considering only the six initial species of the standard protocol (Table 3) that were tested under a larger range of PCR conditions, the highest variation occurred within phylum (Table 2). Urochordates and molluscs, for which we applied the same level of technical effort, display very similar amplification results despite a very different phylogenetic distance relative to species which most influenced primer design. Despite testing fewer primer pairs on the cnidarians, they display only slightly lower numbers of " $\mathrm{P}+\mathrm{I}$ " intron loci than other taxa. Ascidians tend to display small introns (data not shown, available on request), consequently cases of null amplifications due to excessively large fragment size are expected to be less numerous, though this may also lead to reporting the absence of an intron if it is too small ('A' result instead of an 'I or P'). For example, Corella eumyota displays a lower number of successful loci and smaller intron sizes than other ascidians.

The absence of a relationship between global success and phylogenetic position in bilaterians is probably explained by the rules we used to select intron loci before primer design. In fact, the zones selected for the 


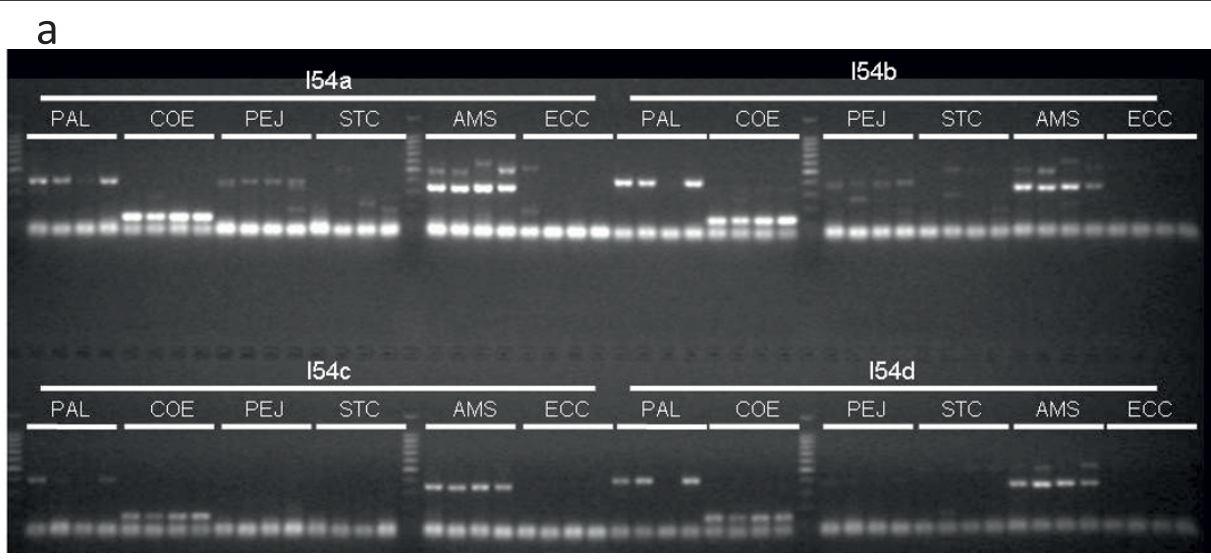

b

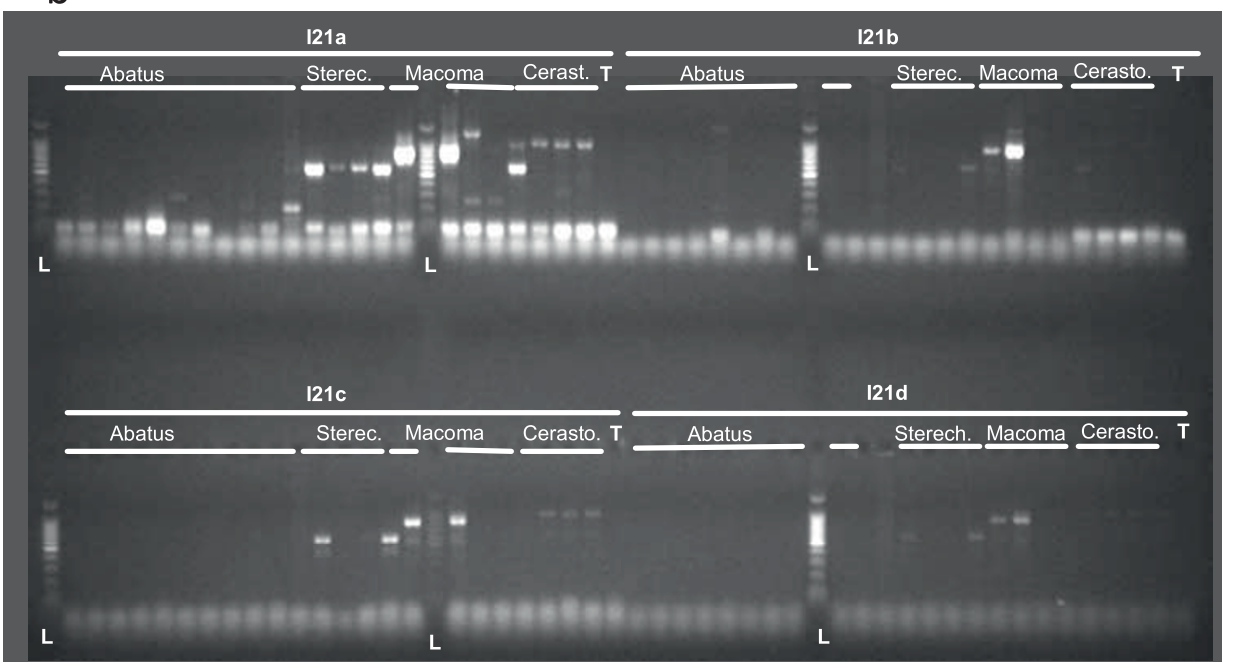

Figure 4 Agarose gel electrophoresis results for 4 primer pairs (one 96-well PCR plate). a: intron 54 (standard protocol); b: intron 21 (S-CR protocol). The size marker, labelled $\mathrm{L}$, is a 100 bp ladder with the brightest band corresponding to $500 \mathrm{bp}$. (a) Four individuals of each species are presented, in the following order: P. lividus, C. eumyota, P. japonica, S. clava, A. squamata, E. cordatum, for intron 54 (primer pairs: a, b, c and d). (b) Lanes successively correspond to the following species (number of individuals in parentheses): Abatus cavernosus (4), A. agassizi (3), A. cordatus (3), A. nimrodi (1), Sterechinus neumayeri (3), S. agassizi (1), Macoma balthica (4), Cerastoderma edule (4).

\section{Table 2 Summary of results for each genus}

\begin{tabular}{lccccc}
\hline Total introns & P & I & A & Nulls & Total \\
\hline Paracentrotus & 15 & 9 & 3 & 25 & 52 \\
Amphipholis & 7 & 23 & 1 & 21 & 52 \\
Echinocardium (all conditions) & 13 & 11 & 1 & 27 & 52 \\
Corella & 7 & 15 & 3 & 27 & 52 \\
Perophora & 15 & 8 & 3 & 26 & 52 \\
Styela & 10 & 14 & 3 & 25 & 52 \\
Abatus (all species) & 14 & 14 & 2 & 22 & 52 \\
Sterechinus (all species) & 12 & 9 & 3 & 28 & 52 \\
Macoma & 6 & 18 & 4 & 24 & 52 \\
Cerastoderma & 11 & 9 & 5 & 27 & 52 \\
Corallium & $6^{*}$ & $12^{*}$ & $5^{*}$ & $6^{*}$ & 29 \\
Paramuricea & $4^{*}$ & $8^{*}$ & $0^{*}$ & $10^{*}$ & 22 \\
\hline
\end{tabular}

Total number of introns in each category of results. *: cnidarians were not tested for all introns.
3' region of the primer design were generally invariant in their amino-acid sequences. Therefore, a good predictor for primer matching may be the similarity of the genome nucleotide composition (influencing codon preference) to the nucleotide composition of the reference species (Ciona, Strongylocentrotus...); since nucleotide composition is variable even at low taxonomic levels (e.g. [20]), the absence of phylogenetic effect is not surprising. The only source of variation is therefore the degeneracy of the code; this is known to vary greatly even within species, so focusing on phylum-specific primers may not be useful when very few taxa are available for a phylum. Actually we have now cloned and sequenced some of these EPICs in echinoderms and we nearly always observe variation within species (even within populations) in the exon sequence (synonymous 


\section{Effort Success}

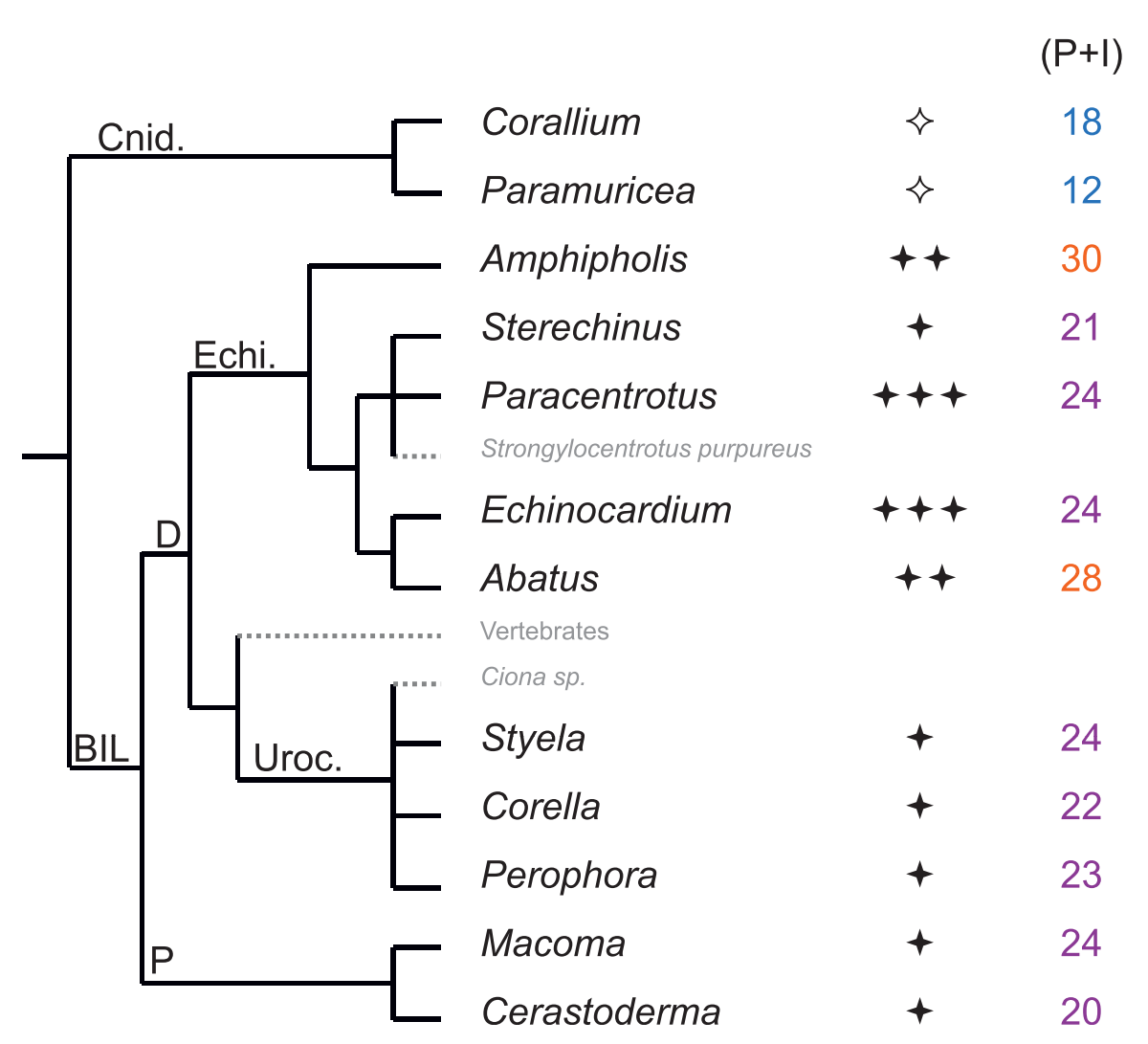

Figure 5 Phylogenetic relationships [39]among the genera tested and global results for intron amplification. To the right of the tested genus (name in black) symbols reflect the level of technical effort [ $\diamond$ Not all primer pairs were tested, $\downarrow$ standard effort, $\downarrow$ more tests than standard (either PCR conditions or DNA extracts), introns scored as ' $P$ ' or ' $I$ '. The taxa whose sequences or genomes most influenced primer design, either by being our models for the nondegenerate part of the codehop primers or by over-representation in gene family databases, are written in small grey letters. Major phylogenetic splits are indicated using the following abbreviations: BIL (Bilateria), P (Protostomia) D (Deuterostomia), Echi. (phylum Echinodermata), Uroc. (phylum Urochordata), Cnid. (phylum Cnidaria).

Table 3 The different protocols and the species and loci (primer pairs) to which they were applied

\begin{tabular}{|c|c|c|c|c|}
\hline $\begin{array}{l}\text { Protocol } \\
\text { name }\end{array}$ & DNA extraction & $\begin{array}{l}\text { PCR } \\
\text { program }\end{array}$ & Primer pairs tested & Species \\
\hline Standard & $\begin{array}{l}\text { Phenol-chloroform (except } P \text {. lividus stage III (i21- } \\
\text { i58) where we used Promega extracts) }\end{array}$ & TD6 & All & $\begin{array}{l}\mathrm{Pl}, \mathrm{As}, \mathrm{Ec}, \mathrm{Ce}, \mathrm{Sc}, \mathrm{Pj}(4 \text { ind. } \\
\text { per species) }\end{array}$ \\
\hline S-F & Idem & Std-Fix & $1,2,3,4,5,6,7,9,13,14,15,17$ & $\begin{array}{l}\mathrm{Pl}, \mathrm{As}, \mathrm{Ec}, \mathrm{Ce}, \mathrm{Sc}, \mathrm{Pj} \text { (4 ind. } \\
\text { per species) }\end{array}$ \\
\hline S-F-60 & Idem & Fix50-60 & $\begin{array}{l}\text { 5b, 21a-d, 25a-d, 35ab, 19ab, 22, 24ab, 29, } \\
\text { 30, 34ab, 49a-d, ATPS } \alpha \text { J, ATPS } \alpha \text { i2 }\end{array}$ & $\begin{array}{l}\mathrm{Pl}, \mathrm{As}, \mathrm{Ec}, \mathrm{Ce}, \mathrm{Sc}, \mathrm{Pj} \text { (4 ind. } \\
\text { per species) }\end{array}$ \\
\hline$S-C R$ & According to taxon and individuals & TD6 & All & $\begin{array}{l}\mathrm{Aca}(4), \mathrm{Aa}(3), \mathrm{Ac}(3), \mathrm{An}(1), \mathrm{Sn} \\
\text { (3), } \mathrm{Sa}(1), \mathrm{Mb}(4), \mathrm{Ce}(4)\end{array}$ \\
\hline EE4 & $\begin{array}{l}1 \text { Qiagen + } 1 \text { Promega + } 1 \text { Chelex + } 1 \text { CTAB- } \\
\text { phenol }\end{array}$ & EE-GP & All & $\mathrm{Ec}(4)$ \\
\hline EE16 & 8 Chelex + 8 Qiagen & EE-GP & $\begin{array}{l}1 \mathrm{~b}, 5 \mathrm{~b}, 22,25,29,37,43,53 \mathrm{~b}, 54 \mathrm{c}, 55, \text { ATP- } \\
\text { Sa, EF4c }\end{array}$ & $\mathrm{Ec}(16)$ \\
\hline GP & 8 Promega & $\mathrm{EE}-\mathrm{GP}$ & $\begin{array}{l}\text { All introns, but only with the primer pair } \\
\text { "a" (Fig. 1) }\end{array}$ & $\mathrm{Pl}(8)$ \\
\hline DA & 3 Qiagen + 1 Phenol-chloroform & DA & $\begin{array}{l}\text { All primer pairs tested for } 29 \text { loci in } \mathrm{Cr}, 22 \\
\text { in Pc (intron numbers in Table 1) }\end{array}$ & $\operatorname{Cr}(3-4), \operatorname{Pc}(3-6)$ \\
\hline
\end{tabular}

The letters $a, b, c$, and $d$ after the intron number refer to primers $F$ and $R, F 2$ and $R, R 2$ and F, F2 and R2 respectively. DA, EE, GP and CR refer to initials of the authors who used them. ind.: individuals. 
changes). However, when the precise pattern at each intron was considered and the data (from Table 1) were analysed through a factorial correspondence analysis, the genera seemed to group according to their phylum (Fig. 6, see legend for details), illustrating the fact that some introns are more useful in some phyla than in others. Within echinoderms, more genera were surveyed, but we did not observe any taxonomic trend, either comparing ophiuroids versus echinoids, or regular versus irregular echinoids. Echinoderms appear widely scattered, by contrast with Urochordates. This may reflect different genome evolutionary rates of those phyla though a richer taxonomic sampling is required to test this hypothesis.

Some gene families appear to be extremely good providers of EPIC markers (Table 4). Remarkably, five introns, from four different gene families, amplify intron-sized products ('P' or 'I') in all the metazoan genera tested (ten to twelve depending on intron) (Table 4). The previously known universal EPICs tested, ATPS and EF1, do not belong to these families.

\section{Effect of primer design}

The least degenerate primers provided the best results (Fig. 7, highly significant exact tests) for the primer pairs composed of two codehop primers (86 cases). This result was not trivial: if the taxa available in our protein alignments had not been sufficiently representative of

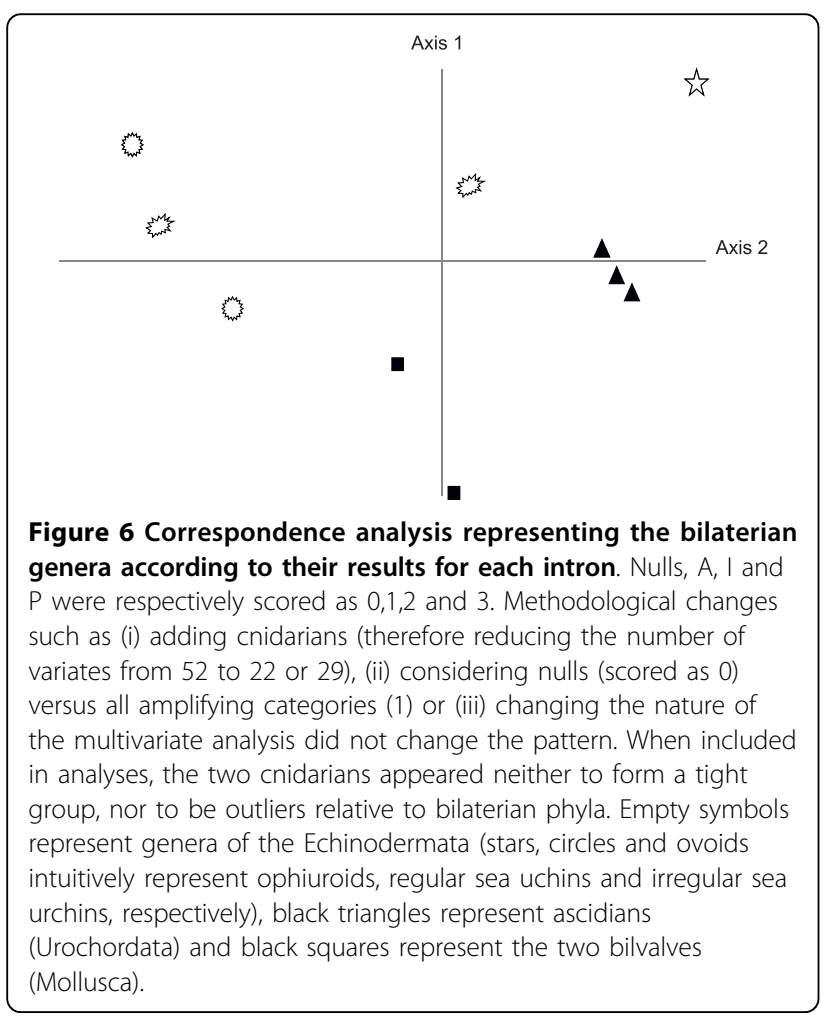

the diversity of phyla tested-among deuterostomes, for instance, the Homolens database provided only vertebrates and one urochordate genus-higher amplification success would have been observed for more degenerate primers. Despite this significant relationship, some of the least degenerate introns at the stage of primer design never amplified (e.g. introns 6, 7, 24 and 49). Codehop primers seem slightly more efficient than primers designed from nucleotide alignments only, though this is not statistically significant (additional file 1).

\section{Effects of PCR program and extraction method}

Comparisons, for 12 primer pairs, of touch-down and fixed annealing temperature programs suggest that touch-down programs are more stringent (less success) and less prone to produce artefactual additional fragments (higher proportion of 'P' patterns) (additional file 1). The two-phase program (tested on 28 primer pairs) also appears to help (additional file 1). However, we obtained no statistical support for these effects. Since in most experiments we used program TD6, our global results may be improved by using alternative programs.

A strong influence of DNA extraction and/or tissue storage history, depending on the species, was revealed (additional file 1).

\section{Obtaining new EPIC loci for any eukaryote lineage}

Our method can potentially be applied to obtain new EPIC loci for any phylogenetic group. Three strategies can be followed. (i) Using the last version of Homolens, the famfetch tool, and the graphical tool developed in this study, one can isolate numerous new EPIC loci, eventually decreasing the stringency level allowing duplication nodes in some genomes, if numerous loci are desired. (ii) More simply, one can focus on the gene families we isolated, and retrieve from protein, nucleotide or EST sequence databases the entries corresponding to the desired lineages; primer pairs should then be designed following our method. (iii) One can just test the primer pairs developed in our study (Fig. 1), preferably using several DNA extraction methods, which can be done in less than 10 working days and for several species simultaneously. Even for kingdoms very distant from metazoans (e.g. plants, fungi, or protists) the second approach may provide successful loci since in some of these gene families the protein alignment files included sequences from plants. We can predict that the success rate will not depend much on the phylogenetic proximity of the taxa to deuterostomes (since those protein sequences are definitely strongly conserved) but will be influenced more on average by the genomic substitution rate of the taxonomic group. For instance, the ecdysozoan phyla (e.g. arthropods and nematodes) may not work as well as molluscs and annelids, though these 
Table 4 Gene families providing best introns and highest numbers of introns

\begin{tabular}{|c|c|c|c|c|c|}
\hline Gene Family & Gene name (from Homolens) & EPIC $n^{\circ}$ & No. bilaterian genera "P or I" & $\mathrm{CR}$ & $\mathrm{PC}$ \\
\hline HBG0011376 & UDP-N-acetylglucosaminyl- & 48 & 9 & NA & NA \\
\hline \multirow[t]{3}{*}{ (Homolens 3) } & transferase & 49 & 0 & 0 & NA \\
\hline & & 50 & 10 & 1 & 1 \\
\hline & & 51 & 9 & 1 & NA \\
\hline HBG0052978 & Ubiquitin & 5 & 10 & 1 & 1 \\
\hline (Homolens 2) & & 9 & 8 & 1 & NA \\
\hline HBG004117 & Calpain & $2=22$ & 10 & 1 & NA \\
\hline (Homolens 2) & & 21 & 10 & 1 & NA \\
\hline \multicolumn{6}{|l|}{$=\mathrm{HBG} 001043$} \\
\hline \multicolumn{6}{|l|}{ (Homolens 3) } \\
\hline HBG0001601 & LD39850p; Peptidylprolyl & 25 & 10 & 1 & 1 \\
\hline \multirow[t]{2}{*}{ (Homolens 3) } & isomerase domain and WD & & & & \\
\hline & repeat-containing protein 1 & & & & \\
\hline HBG0031768 & Glutamyl-prolyl-tRNA- & 52 & 1 & 0 & NA \\
\hline \multirow[t]{4}{*}{ (Homolens 3) } & synthetase & 53 & 7 & 1 & 1 \\
\hline & & 54 & 8 & 1 & 1 \\
\hline & & 55 & 2 & 0 & NA \\
\hline & & 56 & 4 & 0 & 1 \\
\hline HBG0002428 & AT23778p; Colon RCB-0549 Cle- & 29 & 8 & 1 & NA \\
\hline \multirow[t]{2}{*}{ (Homolens 3) } & H3 cDNA; RIKEN full-length & 30 & 4 & NA & NA \\
\hline & enriched library; clone: G430060P17 product:si & 34 & 5 & NA & NA \\
\hline
\end{tabular}

The ten bilaterian genera were tested for all 52 introns, two cnidarian genera were tested for 22 and 29 introns respectively. The number of bilaterian genera displaying promising or intron amplifying patterns ( $\mathrm{P}$ or I) out of the ten genera tested is indicated. The five best introns (10/10 success among bilaterian genera) appear in bold. In the right-hand columns, the results of the two cnidarian genera when tested (not all loci): NA (not available), 1 (promising or intron amplifying), 0 (no intron-size amplicon).CR: Corallium rubrum, PC: Paramuricea clavata.

phyla share a common ancestor relative to deuterostomes, since their genomes are known to evolve rapidly at the nucleotide level $[21,22]$. For the third strategy, based on already defined primers, genome nucleotide composition may be an important factor (see above).

The facts that (i) low degeneracy codehop primers performed better than high degeneracy primers, and (ii) phylogenetic distance (to vertebrates and Ciona) has no relationship with global amplification results, are positive experimental findings of this study, and suggest that the simplest strategy (use of the primers we defined) may be sufficient in most metazoan species to obtain several EPIC loci. In the increasing number of phylogenetic groups where sufficient EST sequence data are available to enrich nucleotide alignments in a variety of taxa, primer design may not require using the Codehop strategy (personal observation from a new ongoing EPIC project).

From EPIC identification to genotyping of large samples Once a promising EPIC locus has been found, it is not always straightforward to directly characterize populations with it. Obtaining sequence data prior to genotyping is recommended. In some cases, good results are provided by direct sequencing of PCR products. There are generally no indels in the exon sequence, so the sequences are readable even with ambiguous positions due to heterozygosity in this region, allowing the design of specific primers. In some (e.g. inbred) species where homozygous individuals are common, direct sequencing is very useful, and eventually, "heterozygous sequences" may be deduced automatically from sequence files containing ambiguities, using dedicated software [23,24]. When direct sequencing is not satisfactory, we recommend cloning the EPIC amplicons from a dozen individuals and sequencing about 10 clones per individual to assess the nature and the level of the variation, permitting (i) the definition of more specific primers if necessary and (ii) the decision whether to characterize alleles by sequence, by size, or by conformation. Some of these introns have been cloned and sequenced in large samples of $P$. lividus, E. cordatum and species not included in the 


\section{Influence of degeneracy level of codehop primers}

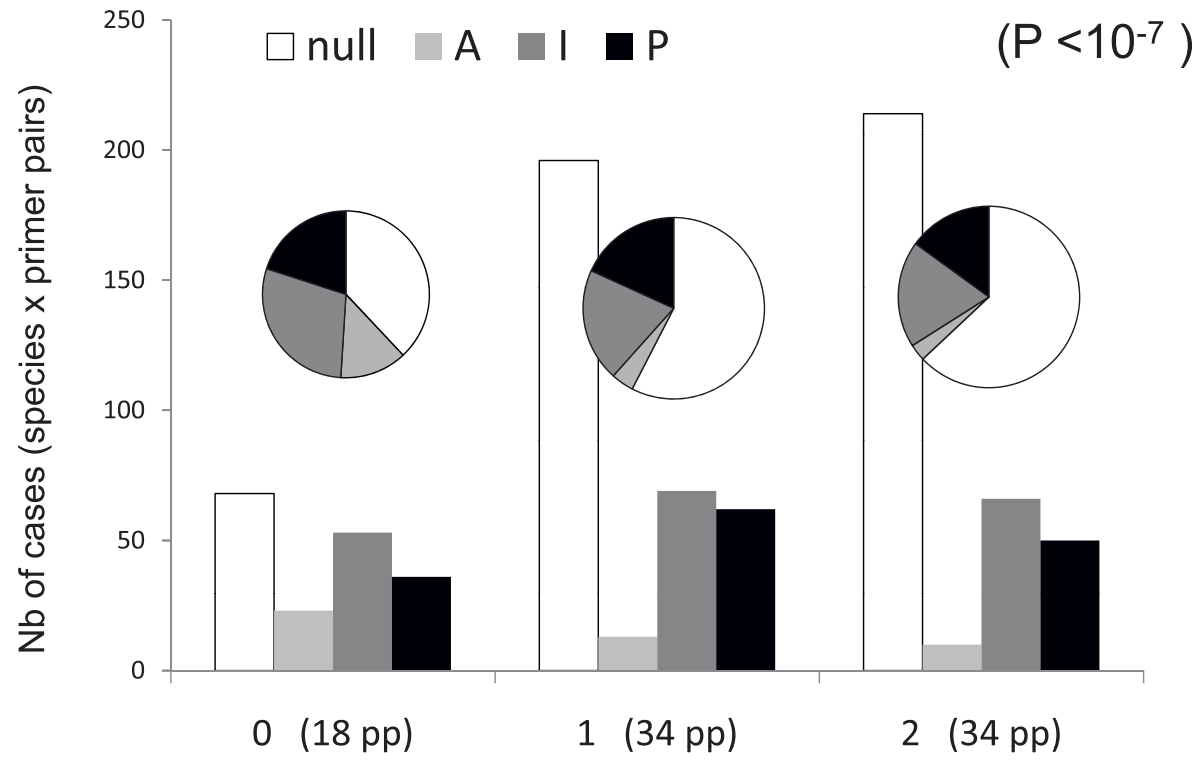

$\mathrm{Nb}$ of primers more than 6-fold degenerate within the pair (number of primer pairs in the category)

Figure 7 Effect of the degeneracy levels of codehop primers on PCR results. Results from the 10 bilaterian genera in which both primers were "codehop" were considered here (they cannot be directly derived from Table 1 since, for some introns, not all primer pairs were "codehop"). The bars represent the total numbers of cases (species $\times$ primer pairs) displaying results ' $P$ ', 'I' ' $A$ ' or nulls, for three categories of introns, those in which no (0), one (1) or both (2) primers have a more than 6 -fold degeneracy. Exact tests were performed from the $4 \times 3$ contingency table used to build the histogram, as well as from tables derived from it after pooling some columns (for instance nulls versus " $\mathrm{A}+$ $\left(+P^{\prime \prime}\right)$ : all were highly significant. The pie chart diagrams display the proportion of the four categories of results within each category of degeneracy; they illustrate the increase in the proportion of null loci when primer degeneracy increases.

present study, and in all cases polymorphism is high due to both indels and substitutions (unpublished). In some cases, the EPIC locus provides diploid Mendelian variation in fragment size visible on agarose gels. Alternatively, finer variations can be revealed using PAGE or automatic sequencers [1]. Conformation techniques (SSCP, DGGE or, more recently, melting curve genotyping (e.g. [25]) allow determination of allele classes, but they have a sensitivity limited to relatively small fragments and they provide no information on allele relationships. The richest information for diploid specimens is a diploid sequence genotype, but classical sequencing techniques do not deal well with heterozygosity. "Next generation sequencing" technologies, such as "454 sequencing", in addition to their extreme rapidity, should allow sequencing a mixture of numerous PCR products, such as for instance from 15 EPIC loci in each of 50 or more individuals, identified by individual labels ( 4 base sequences, inserted in PCR primers or linked to PCR products) for reasonable prices (less than $2000 €$ in 2009). These approaches differ in throughput, cost and sensitivity (Table 5).

\section{Conclusions}

Our new method appears very efficient for finding universal intron loci (EPIC) in sequence data bases whatever the phylum, in metazoans. These EPICs, in addition to providing a set of independent nuclear markers for population genetics and phylogeography, can complement or replace the barcoding molecule used for metazoans, COI, resolving problems associated with single marker studies or inherent in the mitochondrial genome, such as its lack of variability in some phyla [26]. For about ten of these EPICs we obtained sequence data for several individuals, from one to nine distinct genera (unpublished data, available on request). Insertions and deletions were frequent (among which there were a few microsatellites), in only two cases there was no polymorphism, and a minority of cases displayed paralogs or distinct groups of sequences, a problem which was solved when internal primers were defined. This study therefore fills a serious gap in the toolbox of molecular ecologists. These new and universal EPIC loci should generate multilocus sequence datasets from populations of numerous non-model species. Relative to single-locus 
Table 5 Benefits and limits of different genotyping techniques for EPICs (for direct sequencing, see Results-Discussion, last section)

\begin{tabular}{|c|c|c|c|c|c|}
\hline $\begin{array}{l}\text { Genotyping } \\
\text { technique }\end{array}$ & 454 sequencing & Cloning-sequencing & $\begin{array}{l}\text { Melting curve } \\
\text { genotyping }\end{array}$ & SSCP & $\begin{array}{l}\text { Intron length } \\
\text { polymorphism }\end{array}$ \\
\hline $\begin{array}{l}\text { Information (models } \\
\text { of allele evolution) }\end{array}$ & Diploid Seq. (various) & Diploid Seq. (various) & Genotype $\left(\right.$ IAM) $^{i}$ & Genotype $(\text { IAM) })^{i}$ & Genotype (IAM) \\
\hline Fragment size & Seq $<400 \mathrm{bp}^{*}$ & No limit ** & Best $<350$ bp & Best <350 bp & No limit \\
\hline Allele number & No limit & No limit & $\begin{array}{l}\text { Very limited. If high, } \\
\text { impairs genotyping }\end{array}$ & $\begin{array}{l}\text { Some alleles may not } \\
\text { be distinguished }\end{array}$ & $\begin{array}{l}\text { Some alleles may not } \\
\text { be distinguished }\end{array}$ \\
\hline Throughput & high & low & high & Medium & medium \\
\hline PCR & Classical & Classical & Real Time & Classical & Classical \\
\hline Cloning & No & Yes & No & No & No \\
\hline Pooling loci & After careful quantification & $\begin{array}{l}\text { Possible } \# \text { but } \\
\text { problematical }\end{array}$ & No & Possible & Common \\
\hline Electrophoresis & No & For sequencing & No & Yes & Yes \\
\hline Sequencing & $\begin{array}{l}1 \text { run }=\text { Numerous } \\
\text { individuals } \times \text { numerous } \\
\text { loci }\end{array}$ & $\begin{array}{l}\text { Several reactions } \\
\text { required per individual }\end{array}$ & No & No & No \\
\hline $\begin{array}{l}\text { Number human } \\
\text { action steps }{ }^{\mu}\end{array}$ & $L+1+1$ & $\mathrm{~L}+\mathrm{NL}+10 \mathrm{NL}$ & L & $L+L / 3^{\mu^{\prime}}$ & $L+L / 3^{\mu^{\prime}}$ \\
\hline
\end{tabular}

Seq.: sequence. ': IAM: Infinite Allele Model (evolutionary distance is considered identical between all allele pairs). *: Larger amplicons can be included in the run, but only the first $400 \mathrm{bp}$ will be sequenced; however, to favor equal representation among loci, amplicons of relatively uniform lengths (500-900) are preferred. Sequencing can be oriented if desired (start with one of the PCR primers only). This size threshold may increase in future. **: but cloning efficiency may depend on size (and size must be compatible with PCR). \#: not recommended, since loci may be very unequally inserted by cloning vectors (often one missing). ${ }^{\mu}$ : For each technique, the number of steps requiring human action is expressed as a function of the numbers of loci $(\mathrm{L})$ and of genotyped individuals ( $\mathrm{N}$ ) and deduced from the five preceeding rows of the table. We do not consider the possibility of multiplex PCR (i.e. several loci in a single PCR reaction) though this reduces the number of steps). ${ }^{\mu}$ : Pooling three loci together for electrophoresis is generally easy (whether or not an automatic sequencer is used); more loci can be pooled when allelic distributions do not overlap.

sequence data sets or multilocus microsatellite loci datasets, the inferences made from such data using the coalescent theory will be much more precise.

\section{Methods}

\section{Bioinformatic assessment of candidate loci}

We had previously tried a method based on the ExonIntron Database $[26,27,8]$ but this was not successful and thus we developed an original approach. In order to find universal markers from orthologous genes, we took advantage of annotated full-genome sequences to obtain a list of candidate gene families.

The search involved three successive stages (I-III), introducing some methodological changes between stages; each stage contained the following steps, except that step 3 was not performed for stages II-III (see Fig. 2 for flowchart).

\section{Step 1: Gene family selection}

The Famfetch software [28] was used to query revision 2 (stage I of our search) or revision 3 (stages II and III) of the Homolens database of homologous gene families [29] from Ensembl, and retain families for which orthologs were found in Homo, Rodentia, Canis, Gallus, Danio, Xenopus, Percomorpha, Ciona, Insecta, and Nematoda, but no duplication event occurred at the root of any of these taxa in the family tree. This step eliminated known highly conserved families, including most or all of the already known "universal" EPIC loci, but we estimated that the remaining loci would be less prone to paralogy and laboratory effort would be saved. Families in which one isolated species within one of these taxa appeared duplicated were not eliminated (e.g. family HBG001601 was retained (Fig. 3) although Aedes aegyptii presents a duplication, since the duplication is not shared by all Insecta). Step 1 provided 89 gene families for stage I, and 159 gene families for stages II and III, which were merged until step 6 .

\section{Step 2: Protein sequence retrieval}

For each retained gene family, protein sequences were retrieved with a python script, for stage I, or an R script based on the Seqinr package [30], for stages II and III.

\section{Step 3: Enrichment of protein sequence file}

For stage I only, we enriched our protein alignment with protein sequences from other deuterostome species using the Query-Win software to obtain Genbank accession numbers for CDS of deuterostome taxa that were not already included in Homolens. For each gene family, blast searches using the Homo and nematode sequences were performed on this new database; sequences leading to a lower than $10^{-100}$ e-value were considered as homologous sequences. Only 30 additional sequences were identified by this additional step.

\section{Step 4: Multiple-sequence protein alignment}

For each family, a multiple alignment of protein sequences was obtained with ClustalW [31] (stage I) or Muscle [32] (stages II and III). 


\section{Step 5: Intron position identification}

Intron positions and phases were annotated on the multiple alignment using either the Annote_int_mase program (kindly provided by L. Duret, stage I) or an R script using Seqinr (stages II and III).

Step 6 and 7: Retrieval of nucleotide sequences, and choice of introns

Choice of introns occurred before retrieval of nucleotide sequences for stage I and after retrieval for stages II and III. For stage I, we first considered intron positions present in $100 \%$ of all species in all taxonomic groups (as determined by a python script). When such positions were considered promising (embedded within conserved and low-degeneracy amino-acid sequences), we manually retrieved corresponding nucleotide sequences using tblastx, for Strongylocentrotus purpuratus at least (and in some cases a few other species), to determine the $5^{\prime}$ part of the codehop PCR primers (see primer design section).

Stage II was initiated before all stage I families were thoroughly visually screened since we estimated that the method changes introduced in Stage II would save time. For stages II and III, CDS were retrieved and the corresponding nucleotide alignment was obtained using PAL2NAL, for each family. The first 58 protein alignments, taken by increasing Homolens (HBGxxxxxx) number, were examined visually, looking for introns that were present in all the deuterostome species of the alignment and that lay between regions conserved enough to design PCR primers (see below).

In stage III, the remaining 101 families identified from Homolens revision 3 were examined: for this stage, we retrieved potentially homologous sequences from the unannotated genomes of Saccoglossus, Nematostella and Strongylocentrotus, by performing tblastn searches involving the protein sequences of Homo and Ciona. We retained sequences leading to a lower than $10^{-5} \mathrm{e}$-value, and for which protein sequence similarity with some sequences in the original alignment was higher than 0.5. For this stage we developed a new $\mathrm{R}$ script facilitating the visual identification of both intron position and exon sequence conservation in a multiple alignment. This tool allowed us to consider introns whose position varied only slightly among phyla, which may therefore potentially provide EPIC markers (Fig.3). Region conservation was scored by computing a local similarity score $\omega$ in a sliding window along the multiple alignment. This similarity score is the geometric mean of up to four components $\omega_{0} \ldots \omega_{3}$ depending on the number $n$ of species for which additional sequences were available $(1 \leq \mathrm{n} \leq 3)$, where $\omega_{0}$ is the mean local nucleotide similarity between pairs of sequences retrieved from Homolens, and $\omega_{1}, \omega_{2}$ and $\omega_{3}$ are the highest nucleotide identities between pairs of sequences involving one sequence from Homolens and one sequence from Strongylocentrus, Saccoglossus or Nematostella (when sequences from these species were available). We used a sliding window width of 20 nucleotides to compute $\omega$, and identified local peaks of nucleotide similarity in sliding windows of 51 nucleotides width. A plot of nucleotide conservation and intron occurrence was used to visually select promising loci. Contrary to stage I, we did not eliminate potentially usable EPIC loci whose position may vary by a few nucleotides.

In addition to this original research among Homolens families, we specifically examined two genes containing already known "universal" EPIC loci, the Elongation Factor $1 \alpha$ subunit and the ATP-Synthase $\alpha$ subunit $[10,11]$. We retrieved the corresponding metazoan nucleotide sequences from Genbank and aligned them with DIALIGN, specifying that the alignment contained both coding (exon) and non coding regions (introns). Then, we visually examined these alignment files to identify potential EPIC loci.

\section{Primer design}

The great majority of primers were designed using the Codehop method [33,34], which is based on an aminoacid alignment. The $3^{\prime}$ end of the primer (at least 11 bases) is degenerate in a way that represents all possible nucleotide combinations considering that there are usually several codons per amino-acid. The $5^{\prime}$ end, called a clamp, is not degenerate, so that, although it may not anneal during the first two cycles, it should, in the following cycles, perfectly anneal to the primers incorporated and replicated during previous cycles. Such a strategy allows amplification of relatively variable regions, while limiting the problems inherent in highly degenerate primers. Primers were designed only when it was possible to limit degeneracy to less than $32 \times$ (except for a single primer that has $64 \times$ degeneracy) and most of them have $8 \times$ or less degeneracy (Fig. 1). This rule, aimed at reducing the risk of simultaneous amplification of paralogs, eliminated numerous potential loci. In practice, to visually identify conserved regions containing low-degeneracy codons, we used the Bioedit software and customized the amino-acid background color code to reflect the level of codon degeneracy. In some cases when the nucleotide alignment appeared much less variable than predicted from codon degeneracy, conventional degenerate primers were designed, based on the nucleotide alignment (i.e. ambiguities were tolerated along the primer). In these cases, we often decided to design the primer in two parts, with a $3^{\prime}$ part of at least $11 \mathrm{bp}$ degenerate enough to represent all observed nucleotide sequences in our alignment and a nondegenerate $5^{\prime}$ part, mimicking the Codehop design strategy [35]. Below, we will refer to these primers as 
NucHop primers. All details of primer design and sequences are in Fig. 1. The letters a, b, c, and d after the intron number refer to primers pairs $F$ and R, F2 and $\mathrm{R}, \mathrm{R} 2$ and F, F2 and R2 respectively. The design of the $5^{\prime}$ clamp of the primer was based on the Strongylocentrotus nucleotide sequence when available, otherwise on Ciona.

\section{Taxa tested}

Within Bilateria, there are two important clades, the Protostomia, which contain the Lophotrochozoa (such as annelids and molluscs) and the Ecdysozoa (such as arthropods and nematodes), and the Deuterostomia (to which vertebrates, urochordates, and echinoderms belong) (Fig. 5). We first had decided to focus on Deuterostomia in which more sequence data are available, and to survey additional ascidian (Urochordata) species (Corella eumyota, Perophora japonica, and Styela clava) and echinoderm species (regular sea urchins: Paracentrotus lividus, Sterechinus agassizi, Sterechinus neumayeri; spatangoid sea urchins: Echinocardium cordatum, Abatus agassizi, Abatus cavernosus, Abatus cordatus, Abatus ingens, Abatus nimrodi; brittlestars: Amphipholis squamata). To check whether the EPIC loci selected would be exportable to more distant phyla, we also surveyed two mollusc species (Protostomia), Cerastoderma edule and Macoma balthica (both bivalves), and two extremely divergent, non-bilaterian species, the cnidarians Corallium rubrum (the red coral) and Paramuricea clavata. These cnidarians belong to the Octocorallia and are non-symbiotic. Initially, a first round of six species was systematically tested with four individuals per species and the "standard protocol": the three ascidian species, and three echinoderms: P. lividus, E. cordatum, and A. squamata. Then additional tests were made for these echinoderms and additional species. Table 3 reports the number of individuals, loci and species tested per genus, and the corresponding technical conditions.

\section{Molecular methods}

DNA was extracted using phenol-chloroform methods for Paracentrotus lividus and the three ascidian species, plus Echinocardium cordatum, Amphipholis squamata, and Abatus spp. [36]; a saline method was used for Sterechinus spp. [37], and the Nucleon phytopure kit for some Abatus. In addition, specimens of E. cordatum were also extracted using Chelex [38], or with the QIAamp DNA Kit (Qiagen), and specimens of P. lividus were extracted using the Wizard ${ }^{\bullet}$ SV Genomic DNA purification system (Promega). Paramuricea clavata DNA was extracted using the QIAamp ${ }^{\circ}$ kit, and we used standard phenol-chloroform extractions for Corallium rubrum. Polymerase Chain Reactions were performed in a $10 \mu \mathrm{l}$ final volume, using $0.25 \mathrm{u}$ of $\mathrm{GoTaq}^{\circ}$ Flexi-DNA Polymerase and green buffer (Promega), in $5 \mathrm{mM}$ $\mathrm{MgCl}_{2}$, and $0.2 \mathrm{mM} \mathrm{dNTP}$, with 10 pmol of each primer, and with $10 \mathrm{ng}$ of template DNA, except for cnidarians for which template DNA probably exceeded $10 \mathrm{ng}$. PCR cycling temperature programs with fixed annealing temperatures (Table 6) were first tried for a few EPIC loci (trials at $48,50,55$, or $60^{\circ} \mathrm{C}$, according to locus referred as "St-fix" or "fix-50-60" in Table 6), and compared with two touch-down programs (TD6, TD6'); subsequently, touch-down programs were preferred and were used for all primer pairs for all EPIC loci except cnidarians (referred as the standard protocol, Table 3). For $E$. cordatum and P. lividus, all loci were additionally amplified with fixed annealing temperatures (named EEGP in Table1). For cnidarians, a similar program was used at $45^{\circ} \mathrm{C}$ (DA in Table 6). PCR products were visualized by electophoresis using $5 \mu \mathrm{l}$ of product on a $1 \mathrm{X}$ TBE-1.5\% agarose gel $(260 \mathrm{ml})$ in a Biorad SubCell Model 192 Cell, gels of $25 \times 25 \mathrm{~cm}$ and 4 combs of 51 wells, during ca $1 \mathrm{~h}$ migration at $120 \mathrm{~V}$, using 2.5 to 5 $\mu \mathrm{l}$ of $100 \mathrm{bp}$ Benchtop ladder ${ }^{\bullet}$ (Promega) as size marker.

\section{Nomenclature used to characterize the results to allow comparison of methods}

Results of PCR amplification as observed from agarose gels were characterized, at first on a per species basis, using the following rules. 'Promising' results (' $\mathrm{P}$ ' in Table 1) refer to the observation in all individuals of the species of amplification products that were not too faint, did not display numerous fragments (rare cases with

Table 6 Description of PCR programs

\begin{tabular}{ll}
\hline Program & Description \\
\hline Std-Fix & $3^{\prime} 94^{\circ} ; 35 \times\left[40^{\prime \prime} 94^{\circ} \mathrm{C} ; 1^{\prime}\left(45,48,50\right.\right.$, or $55^{\circ} \mathrm{C}$ according to experiment), $\left.2^{\prime} 72^{\circ} \mathrm{C}\right], 3^{\prime} 72^{\circ} \mathrm{C}$ \\
Fix $50-60^{\circ} \mathrm{C}$ & $3^{\prime} 94^{\circ} ; 8 \times\left[30^{\prime \prime} 94^{\circ} \mathrm{C} ; 1^{\prime} 50^{\circ} \mathrm{C}, 1^{\prime} 72^{\circ}, 26 \times\left[30^{\prime \prime} 94^{\circ} \mathrm{C} ; 30^{\prime \prime} 60^{\circ} \mathrm{C} ; 1^{\prime} 72^{\circ} \mathrm{C}\right], 3^{\prime} 72^{\circ} \mathrm{C}\right.$ \\
TD6 & $2^{\prime} 94^{\circ} \mathrm{C} ; 14 \times\left[1^{\prime} 94^{\circ} \mathrm{C}, 1^{\prime} 58^{\circ} \mathrm{C}\right.$ to $\left.45^{\circ} \mathrm{C}\left(-1^{\circ} \mathrm{C} / \mathrm{Cycle}\right), 1^{\prime} 72^{\circ} \mathrm{C}\right], 25 \times\left[30^{\prime \prime} 94^{\circ} \mathrm{C}, 45^{\prime \prime} 58^{\circ} \mathrm{C}, 45^{\prime \prime} 72^{\circ} \mathrm{C}\right], 3^{\prime} 72^{\circ} \mathrm{C}$ \\
TD6 & $2^{\prime} 94^{\circ} \mathrm{C} ; 14 \times\left[1^{\prime} 94^{\circ} \mathrm{C}, 1^{\prime} 58^{\circ} \mathrm{C}\right.$ to $\left.45^{\circ} \mathrm{C}\left(-1^{\circ} \mathrm{C} / \mathrm{Cycle}\right), 1^{\prime} 72^{\circ} \mathrm{C}\right], 25 \times\left[40^{\prime \prime} 94^{\circ} \mathrm{C}, 40^{\prime \prime} 58^{\circ} \mathrm{C}, 1^{\prime} 72^{\circ} \mathrm{C}\right], 3^{\prime} 72^{\circ} \mathrm{C}$ \\
EE-GP & $2^{\prime} 94^{\circ} ; 35 \times\left[20^{\prime \prime} 94^{\circ} \mathrm{C} ; 1^{\prime} 45^{\circ} \mathrm{C} ; 1^{\prime} 72^{\circ}\right] ; 5^{\prime} 72^{\circ} \mathrm{C}$ \\
DA & $3^{\prime} 94^{\circ} \mathrm{C} ; 30 \times\left[1^{\prime} 94^{\circ} \mathrm{C} ; 1^{\prime} 45^{\circ} \mathrm{C} ; 1^{\prime} 72^{\circ} \mathrm{C}\right] ; 5^{\prime} 72^{\circ} \mathrm{C}$ \\
\hline
\end{tabular}

$\mathrm{DA}, \mathrm{EE}$ and GP refer to initials of the authors who used them. ': minute. ": second. 
three bands were admitted, when one was constant) and were long enough to contain an intron of $c a 70-100 \mathrm{bp}$ at least. The second category is designated 'I' (intronsize amplicon), and corresponds to amplicons of the size of an intron that were very faint or displayed multiple bands or failed to amplify in at least one individual. The third category, 'Amplification' (designated 'A'), corresponds to amplification products too short to contain a useful intron. Results in the subsequent tables and text are reported by genus after pooling all the protocols that were tried. For the species tested with several protocols (P. lividus, E. cordatum), and for the genera where several species were tested (i.e. Abatus, Sterechi$n u s)$, the best result is reported.

\section{Additional material}

Additional file 1: Influence of molecular biology methods on EPIC amplification success. It contains three paragraphs: (1) Are the codehop primers more efficient than primers designed from nucleotide alignments only? (2) Effect of PCR programs, and (3) Effect of DNA extraction and tissue conservation history.

\section{Abbreviations}

CDS: Coding DNA Sequence; EPIC: Exon Primed Intron Crossing; PCR: Polymerase Chain Reaction; SSCP: Single Strand Conformation Polymorphism; DGGE: Denaturing Gradient Gel Electrophoresis; PAGE: PolyAcrylamide Gel Electrophoresis.

\section{Acknowledgements}

We thank Paolo Sordino who accepted this project in the workpackage he co-coordinated with F. P. Patti, within the European network of excellence "Marine Genomics Europe" (GOCE-CT-2004-505403), providing funds for consumables and TH's salary. We also thank Laurent Duret for helpful suggestions, Jeanine Olsen and Jean-Pierre Féral for stimulating the extension of the study to molluscs. PCL was supported by NWO-Meervoud and ESF Conservation Genetics grants.

\section{Author details \\ ${ }^{1}$ African Coelacanth Ecosystem Programme, Department of Genetics, University of Pretoria, Lynnwood Road 0002, Pretoria, South Africa. ${ }^{2}$ Marine Biological Association of the UK, The Laboratory, Citadel Hill Plymouth, PL1 2PB, UK. ${ }^{3}$ Department of Genetics, Forestry and Agricultural Biotechnology Institute (FABI), University of Pretoria, Pretoria, South Africa. ${ }^{4}$ Instituto de Ecología y Biodiversidad, Departamento de Ciencias Ecológicas, Facultad de Ciencias, Universidad de Chile, Santiago, Chile. ${ }^{5}$ Alfred Wegener Institute for Polar and Marine Research, Wadden Sea, Station Sylt, Hafenstr. 43, 25992 List, Germany. ${ }^{6}$ Royal Netherlands Institute for Sea Research, Department of Marine Ecology, P.O. Box 59, 1790AB Den Burg, The Netherlands. 'Functional and Evolutionary Ecology Laboratory, Stazione Zoologica "Anton Dohrn", Villa Dohrn, Punta S. Pietro, Napoli, Italia - 80077 Ischia (Naples), Italy. ${ }^{8}$ Plant Ecological Genetics Group, Institute of Integrative Biology, ETH Zurich, Universitätsstrasse 16, CHN, 8092 Zurich, Switzerland. ${ }^{9}$ Université de Lyon, F- 69000, Lyon, France. ${ }^{10}$ Université Lyon 1; CNRS, UMR5558, Laboratoire de Biométrie et Biologie Evolutive, F-69622, Villeurbanne, France. ${ }^{11}$ Aix-Marseille Université, Laboratoire DIMAR (diversité, évolution et écologie fonctionnelle marine), CNRS UMR6540, rue de la batterie des Lions, 13007 Marseille, France.}

\section{Authors' contributions}

TH performed the majority of laboratory work, and designed primers. NB and SM developed all the dedicated bioinformatic tools. CR performed the laboratory work for several species; GP, EE, DA and KMJ tested all/numerous primer pairs for one species, and EE, in addition, compared DNA extraction methods. AC conceived and supervised the project, selected loci and designed primers, and wrote the article. Other authors provided DNA extracts. Some authors in addition improved the manuscript. All authors read and approved the final manuscript.

Received: 1 April 2010 Accepted: 13 September 2010 Published: 13 September 2010

\section{References}

1. Chenuil $A$ : Choosing the right molecular genetic markers for studying biodiversity: from molecular evolution to practical aspects. Genetica 2006, 127:101-120.

2. Anmarkrud JA, Kleven $O$, Bachmann L, Lifjeld JT: Microsatellite evolution: Mutations, sequence variation, and homoplasy in the hypervariable avian microsatellite locus HrU10. BMC Evolutionary Biology 2008, 8:138.

3. Arcot SS, Wang ZY, Weber JL, Deininger PL, Batzer MA: Alu Repeats - a source for the genesis of primate microsatellites. Genomics 1995, 29(1):136-144.

4. Grillo V, Jackson F, Gilleard JS: Characterisation of Teladorsagia circumcincta microsatellites and their development as population genetic markers. Molecular and Biochemical Parasitology 2006, 148(2):181-189.

5. Jayashree B, Jagadeesh VT, Hoisington D: CISprimerTOOL: software to implement a comparative genomics strategy for the development of conserved intron scanning (CIS) markers. Molecular Ecology Notes 2007, 8(3):575-577

6. Bierne N, Lehnert A, Bedier E, Bonhomme F, Moore SS: Screening for intron-length polymorphisms in penaeid shrimps using exon-primed intron-crossing (EPIC)-PCR. Molecular Ecology 2000, 9(2):233-235.

7. Li C, Riethoven J-JM, Ma L: Exon-primed intron-crossing (EPIC) markers for non-model teleost fishes. BMC Evolutionary Biology 2010, 10:90.

8. Fedorov A, Merican AF, Gilbert W: Large-scale comparison of intron positions among animal, plant and fungal genes. Proc Natl Acad Sci USA 2002, 99(25):16128-16133.

9. Putnam NH, Srivastava M, Hellsten U, Dirks B, Chapman J, Salamov A, Terry A, Shapiro H, Lindquist E, Kapitonov W, et al: Sea anemone genome reveals ancestral eumetazoan gene repertoire and genomic organization. Science 2007, 317(5834):86-94.

10. Palumbi SR: Nucleic Acids II. Chapter 7: The polymerase chain reaction. In Molecular systematics. Edited by: Hillis DM, Moritz C, Mable BK. Massachussetts: Sinauer. Sunderland; 1996:205-247.

11. Jarman SN, Ward RD, Elliott NG: Oligonucleotide primers for PCR amplification of coelomate introns. Mar Biotechnol 2002, 4:347-355.

12. Concepcion GT, Crepeau MW, Wagner D, Kahng SE, Toonen RJ: An alternative to ITS, a hypervariable, single-copy nuclear intron in corals, and its use in detecting cryptic species within the octocoral genus Carijoa. Coral Reefs 2008, 27(2):323-336.

13. Cordero D, Pena JB, Saavedra C: Polymorphisms at three introns in the Manila clam (Ruditapes philippinarum) and the grooved carpet-shell clam (R-decussatus). Journal of Shellfish Research 2008, 27(2):301-306.

14. Foltz DW: An ancient repeat sequence in the ATP synthase beta-subunit gene of forcipulate sea stars. Journal of Molecular Evolution 2007, 65(5):564-573.

15. Boissin E, Feral JP, Chenuil A: Defining reproductively isolated units in a cryptic and syntopic species complex using mitochondrial and nuclear markers: the brooding brittle star, Amphipholis squamata (Ophiuroidea). Molecular Ecology 2008, 17(7):1732-1744.

16. Daguin C, Bonhomme F, Borsa P: The zone of sympatry and hybridization of Mytilus edulis and M. galloprovincialis, as described by intron length polymorphism at locus mac-1. Heredity 2001, 86:342-354.

17. Ohresser $M$, Borsa $P$, Delsert $C$ : Intron-length polymorphism at the actin locus Mac-1: a genetic marker for population studies in the marine mussels Mytilus galloprovincialis Lmk. and M. edulis L. Mol Mar Biol Biotechnol 1997, 6(2):123-130.

18. Hassan M, Lemaire C, Fauvelot C, Bonhomme F: Seventeen new exonprimed intron-crossing polymerase chain reaction amplifiable introns in fish. Molecular Ecology Notes 2002, 2(3):334-340.

19. Atarhouch T, Rami M, Cattaneo-Berrebi G, Ibanez C, Augros S, Boissin E, Dakkak A, Berrebi P: Primers for EPIC amplification of intron sequences 
for fish and other vertebrate population genetic studies. Biotechniques 2003, 35(4):676-682.

20. Mitreva M, Wendl MC, Martin J, Wylie T, Yin Y, Larson A, Parkinson J, Waterston RH, McCarter JP: Codon usage patterns in Nematoda: analysis based on over 25 million codons in thirty-two species. Genome Biology 2006, 7(8)

21. Raible F, Tessmar-Raible K, Osoegawa K, Wincker P, Jubin C, Balavoine G Ferrier D, Benes V, de Jong P, Weissenbach J, et al: Vertebrate-type intronrich genes in the marine annelid Platynereis dumerilii. Science 2005, 310(5752):1325-1326

22. Zimek $\mathrm{A}$, Weber $\mathrm{K}$ : In contrast to the nematode and fruit fly all 9 intron positions of the sea anemone lamin gene are conserved in human lamin genes. European Journal of Cell Biology 2008, 87(5):305-309.

23. Stephens $M$, Scheet $P$ : Accounting for decay of linkage disequilibrium in haplotype inference and missing-data imputation. American Journal of Human Genetics 2005, 76(3):449-462.

24. Dixon CJ: OLFinder:a program which disentangles DNA sequences containing heterozygous indels. Molecular Ecology Resources 2010, 10(2):335-340.

25. Ye J, Parra EJ, Sosnoski DM, Hiester K, Underhill PA, Shriver MD: Melting curve SNP(McSNP) Genotyping: a useful approach for diallelic genotyping in forensic science. Journal of Forensci Science 2002, 47(3):593-600.

26. Van der Ham JL, Brugler MR, France SC: Exploring the utility of an indelrich, mitochondrial intergenic region as a molecular barcode for bamboo corals (Octocorallia: Isididae). Marine Genomics 2009, 2(34):183-192.

27. Saxonov S, Daizadeh I, Fedorov A, Gilbert W: EID: The Exon Intron Database-an exhaustive database of protein-coding intron-containing genes. Nucleic Acids Res 2000, 28(1):185-190

28. Dufayard JF, Duret L, Penel $S$, Gouy M, Rechenmann F, Perrière G: Tree pattern matching in phylogenetic trees: automatic search for orthologs or paralogs in homologous gene sequence databases. Bioinformatics 2005, 21:2596-2603.

29. Penel S, Arigon AM, Dufayard JF, Sertier AS, Daubin V, Duret L, Gouy M, Perrière G: Databases of homologous gene families for comparative genomics. BMC Bioinformatics 2009, 10(Suppl 6):S3.

30. Charif D, Lobry J, (eds): SeqinR 1.0-2: a contributed package to the R project for statistical computing devoted to biological sequences retrieval and analysis. 2007.

31. Thompson JD, Higgins DG, Gibson TJ: CLUSTAL-W - Improving the sensitivity of progressive multiple sequence alignment through sequence weighting, position specific gap penalties and weight matrix choice. Nucleic Acids Research 1994, 22(22):4673-4680.

32. Edgar RC: MUSCLE: a multiple sequence alignment method with reduced time and space complexity. Bmc Bioinformatics 2004, 5:1-19.

33. Rose TM, Henikoff JG, Henikoff S: CODEHOP (COnsensus-DEgenerate Hybrid Oligonucleotide Primer) PCR primer design. Nucleic Acids Res 2003, 31(13):3763-3766.

34. Rose TM, Schultz ER, Henikoff JG, Pietrokovski S, McCallum CM, Henikoff S: Consensus-Degenerate hybrid oligonucleotide primers for amplification of distantly related sequences. Nucleic Acids Res 1998, 26(7):1628-1635.

35. Hoareau TB, Boissin E: Design of phylum-specific hybrid primers for DNA barcoding: addressing the need for efficient $\mathrm{COI}$ amplification in the Echinodermata. Molecular Ecology Resources 2010, 9999(9999).

36. Winnepenninckx B, Backeljau T, De Wachter R: Extraction of high molecular weight DNA for molluscs. Trends in Genetics 1993, 9(12):407.

37. Aljanabi SM, Martinez I: Universal and rapid salt-extraction of high quality genomic DNA for PCR-based techniques. Nucleic Acids Research 1997, 25(22):4692-4693.

38. Walsh PS, Metzger DA, Higushi R: Chelex 100 as a medium for simple extraction of DNA for PCR-based typing from forensic material. Biotechniques 1991, 10(4):506-513.

39. Lecointre G, Le H: Classification phylogénétique du vivant. Paris: Belin 2001.

doi:10.1186/1471-2148-10-276

Cite this article as: Chenuil et al: An efficient method to find potentially universal population genetic markers, applied to metazoans. BMC Evolutionary Biology 2010 10:276.

\section{Submit your next manuscript to BioMed Central and take full advantage of:}

- Convenient online submission

- Thorough peer review

- No space constraints or color figure charges

- Immediate publication on acceptance

- Inclusion in PubMed, CAS, Scopus and Google Scholar

- Research which is freely available for redistribution 